\title{
A Controvérsia do Uso dos Testes Psicológicos por Psicólogos e Não Psicólogos
}

\author{
Denise Ruschel Bandeira ${ }^{1}$ \\ ${ }^{1}$ Universidade Federal do Rio Grande do Sul, RS, Brasil.
}

Resumo: Nos últimos anos, no Brasil, tem-se discutido o uso de testes psicológicos por outros profissionais. Essa é uma questão que se tornou complexa especificamente no Brasil tendo em vista as características da nossa formação em Psicologia assim como as legislações da área. Com o objetivo de contribuir para essa discussão, apresento os fatores que entendo como necessários de serem pensados: formação, avaliação dos instrumentos, certificação para uso dos testes e fiscalização. A partir de uma visão das características da nossa formação, dos processos de avaliação de testes no Brasil e no mundo, proponho possibilidades de certificação e fiscalização de forma que seja possibilitado o uso de instrumentos a quem tenha formação e qualificação para isso e não somente um registro profissional no Conselho Federal de Psicologia.

Palavras-chave: Uso de Testes Psicológicos, Formação em Psicologia, Avaliação de Testes Psicológicos.

\section{The Controversy of the Use of Psychological Tests by Psychologists and Non Psychologists}

Abstract: Recently, in Brazil, the use of psychological tests by other professionals has been discussed. This issue has become complex especially in our country due to the characteristics of our training in Psychology as well as the laws of the area. In order to contribute to this discussion, I present the factors that I think are necessary to be considered: training, review of instruments, certification and control for the use of tests. Addressing the characteristics of our psychology training, test review processes in Brazil and in the world, I propose possibilities of certification and control for the use of instruments, so that their use is possible to those who have training and qualification for it, not just a registration number in the Professional Council of Psychologist.

Keywords: Use of Psychological Tests, Training in Psychology, Review of Psychological Tests.

\section{La Controversia del Uso de las Pruebas Psicológicas por Psicólogos y No Psicólogos}

Resumen: En los últimos años, en Brasil, se ha discutido el uso de pruebas psicológicas por otros profesionales. Esta es una cuestión que se ha vuelto compleja específicamente en nuestro país teniendo en cuenta las características de nuestra formación en Psicología así como las legislaciones del área. Con el objetivo de contribuir a esta discusión, presento los factores que entiendo como necesarios de ser pensados: formación, evaluación de los instrumentos, certificación para uso de las pruebas y fiscalización. A partir de una visión de las características de nuestra formación, de los procesos de evaluación de pruebas en Brasil y en el mundo, propongo posibilidades de certificación y fiscalización de forma que sea posibilitado el uso de instrumentos a quienes tenga formación y calificación para ello, no sólo un registro profesional en el Consejo Federal de Psicología.

Palabras clave: Uso de Pruebas Psicológicas, Formación en Psicología, Evaluación de Pruebas Psicológicas. 
Quem é que pode utilizar os chamados testes psicológicos? Uma resposta simples a essa pergunta seria: se são testes psicológicos, somente os psicólogos. Para isso, precisamos então definir o que são testes psicológicos. Resposta superficial: aqueles que avaliam construtos psicológicos, tais como inteligência, personalidade, memória, atenção e tantos outros. A questão é que esses mesmos construtos são estudados e avaliados por outras áreas, cada uma com sua "lente". Os testes são somente instrumentos que trazem dados sobre pessoas; e os profissionais, de acordo com sua formação, vão entender esses dados do ponto de vista dos conhecimentos construídos na sua formação. O que devemos objetivar é um uso ético e responsável dos instrumentos de avaliação.

Para contribuir de modo mais profundo nessa controvérsia e tentar responder à questão de quem pode usar testes psicológicos, entendo que são vários os fatores que devem ser considerados. Vou elencar alguns sobre os quais pretendo discorrer neste artigo: formação, avaliação dos instrumentos, certificação para uso dos testes e fiscalização.

\section{Formação}

Antes de iniciar essa discussão, é importante que fiquem claros os conceitos de teste e de avaliação. Conforme Urbina (2014), teste psicológico é um procedimento sistemático que tem como objetivo coletar amostras de comportamento de um indivíduo, que podem ser de caráter cognitivo, afetivo ou interpessoal. Caracteriza-se como um instrumento que avalia construtos não observados diretamente (Hutz, 2015). O seu uso implica treinamento específico, com conhecimento a respeito do construto avaliado. Avaliação, conforme Resolução CFP no 09/2018 (p. 2), é

um processo estruturado de investigação de fenômenos psicológicos, composto de métodos, técnicas e instrumentos, com o objetivo de prover informações à tomada de decisão, no âmbito individual, grupal ou institucional, com base em demandas, condições e finalidades específicas.

Portanto, para se realizar uma avaliação (e aqui focando em avaliação psicológica de pessoas), pressupõe-se que o profissional tenha conhecimentos gerais obtidos durante uma formação ideal em cursos de Psicologia, tais como Psicologia do Desenvolvimento, Psi- cologia da Personalidade, Psicopatologia, Avaliação Psicológica, Psicometria, Técnicas de Entrevista, Pesquisa em Psicologia, Psicologia Clínica, Neuropsicologia, entre outras (Bandeira, Trentini, \& Krug, 2016). Ainda, se considerarmos áreas específicas tais como Forense ou Organizacional, por exemplo, mais disciplinas são necessárias.

Ainda, numa avaliação psicológica, o psicólogo precisará se preocupar com o contexto do sujeito. Necessitará também conhecer os testes que utilizará, ou melhor, possuir treinamento específico. Quando não possuir, que tenha condições, a partir de seu conhecimento prévio sobre o construto avaliado e de noções de Psicometria, aprender o teste por meio do seu manual. Com tudo isso quero dizer que o questionamento a ser tratado neste artigo não recai sobre o quanto a avaliação psicológica é de uso exclusivo do psicólogo. Para esta, não há dúvida! E, sim, sobre o quanto os testes psicológicos devem ser de uso exclusivo do psicólogo.

Com relação aos testes psicológicos, atualmente no Brasil, todo psicólogo formado em cinco anos de curso pode usar testes psicológicos. Independentemente do curso que ele fez, das disciplinas que cursou, ele está autorizado pelo Conselho Federal de Psicologia (CFP) a utilizar testes. Então me pergunto, o quanto todo psicólogo pode usar os testes? O quanto todo psicólogo fez uma formação que o capacite para tanto? Ou mesmo, o quanto está capacitado para realizar uma avaliação? Então, precisamos falar sobre formação. Iniciarei com algumas ideias já escritas e pensadas que questionam como é a formação em Psicologia em nosso país (Alchieri \& Bandeira, 2002; Bandeira, 2011; Hutz \& Bandeira, 2003). No Brasil, o estudante de Psicologia, após a Resolução no 5 (2011), que instituiu as diretrizes curriculares para os cursos de graduação em Psicologia, passou a ter que escolher uma (ou duas) ênfases em seu curso. Isso significa que se $o$ aluno não escolhe uma ênfase na qual tenha conhecimentos aprofundados para o trabalho com instrumentos de avaliação psicológica, sua formação será muito básica, por vezes até pífia, para essa área.

Essa resolução estabeleceu uma série de competências e habilidades esperadas na formação em Psicologia, a serem adquiridas durante o núcleo básico do curso, ou seja, em três anos. Listo aqui algumas das quais são mais ligadas à área de avaliação psicológica (Resolução nº 05/2011):

[...] III - identificar e analisar necessidades de natureza psicológica, diagnosticar, elaborar projetos, 
planejar e agir de forma coerente com referenciais teóricos e características da população-alvo; IV identificar, definir e formular questões de investigação científica no campo da Psicologia, vinculando-as a decisões metodológicas quanto à escolha, coleta e análise de dados em projetos de pesquisa; $\mathrm{V}$ - escolher e utilizar instrumentos e procedimentos de coleta de dados em Psicologia, tendo em vista a sua pertinência; VI - avaliar fenômenos humanos de ordem cognitiva, comportamental e afetiva, em diferentes contextos; VII - realizar diagnóstico e avaliação de processos psicológicos de indivíduos, de grupos e de organizações; [...] IX - atuar inter e multiprofissionalmente, sempre que a compreensão dos processos e fenômenos envolvidos assim o recomendar; $\mathrm{X}$ - relacionar-se com o outro de modo a propiciar o desenvolvimento de vínculos interpessoais requeridos na sua atuação profissional; [...] XIII - elaborar relatos científicos, pareceres técnicos, laudos e outras comunicações profissionais, inclusive materiais de divulgação; [...] XV - saber buscar e usar o conhecimento científico necessário à atuação profissional, assim como gerar conhecimento a partir da prática profissional (p. 3).

Quem é professor de graduação em Psicologia no Brasil, sabe muito bem que essas competências e habilidades podem ser adquiridas, em parte, durante o núcleo básico do curso, mas que devem ser aprofundadas na ênfase. Então, quando um aluno escolhe uma ênfase na qual ele poderá conhecer de forma mais aprofundada e crítica alguns instrumentos de avaliação e formar um conceito mais completo para poder avaliar melhor os instrumentos, ele terá condições de escolher com maior grau de certeza o que deve ou não utilizar em seus processos de avaliação (lendo e entendendo manuais e artigos científicos que mostram os avanços da área). Além disso, terá consciência da necessidade de se manter atualizado, pois não se consegue aprender todos os instrumentos disponíveis no mercado (atualmente, junho de 2016, o Sistema de Avaliação de Testes Psicológicos - Satepsi - conta com 169 testes favoráveis na sua lista, ver em http://satepsi.cfp.org.br/).

Porém, quando um aluno não se interessa pela área de avaliação, a tendência é que ele não forme essa base de conhecimento. Contudo, sendo ele um psicólogo, ele está autorizado pelo CFP a utilizar testes psicológicos e realizar processos de avaliação psi- cológica. Lembrando, a Lei ${ }^{\circ} 4.119$, que regulamenta a profissão do psicólogo diz que:

$\S 1^{\circ}$ - Constitui função privativa do Psicólogo a utilização de métodos e técnicas psicológicas com os seguintes objetivos: a) diagnóstico psicológico; b) orientação e seleção profissional; c) orientação psicopedagógica; d) solução de problemas de ajustamento.

Portanto, entendo que aqui temos o primeiro problema. Quando a lei foi instituída, os cursos tinham uma grande dedicação para o ensino de métodos e técnicas psicológicas para estes quatro objetivos. Após a Resolução no 5 de 2011, o aluno passou a escolher sua ênfase e não necessariamente sua formação abrange tudo isso. Ora, se os testes psicológicos são de uso exclusivo do psicólogo e somente ele é autorizado a utilizá-los, não teria que ser a maior parte do curso destinada a esses conhecimentos? Só que não é essa a realidade, nem é isso que queremos da Psicologia no Brasil. Seria resumi-la a muito pouco. Podemos pensar que a criação do título de especialista em avaliação psicológica pelo CFP seria uma saída, e que somente as pessoas que fizessem uma formação focada nesta área, receberiam essa possibilidade de atuação, de uso exclusivo do psicólogo.

\section{Avaliação dos instrumentos}

Na controvérsia do uso de instrumentos psicológicos por não psicólogos, outro aspecto a ser considerado é o quanto a avaliação de instrumentos psicológicos deve ser realizada por psicólogos. Considerando que para isso são necessários conhecimentos de Psicometria e Avaliação Psicológica, é muito coerente que essa seja uma atividade de psicólogos que obtiveram uma formação aprofundada na área.

$\mathrm{Na}$ avaliação de instrumentos, entendo que nossa área está se saindo muito bem pois nossos instrumentos são avaliados com critérios rigorosos. $\mathrm{O}$ processo de revisão e avaliação dos instrumentos teve início em 1997, com a instituição da Câmara Interinstitucional de Avaliação Psicológica, pelo CFP. Um pouco dessa história pode ser conhecido em publicações do CFP, a saber, Avaliação Psicológica: Diretrizes na Regulamentação da Profissão (CFP, 2010), no texto de Anache e Corrêa (2010) e Ano da Avaliação Psicológica - Textos Geradores (CFP, 2011), em especial, o texto de Santos (2011). 
Desde lá, muitos foram os avanços e tropeços, mas hoje podemos dizer que nosso processo avaliativo é de qualidade, representado pelo Satepsi (ver em http://satepsi.cfp.org.br/). Os testes submetidos ao Satepsi são avaliados por pelo menos dois experts na área do teste e posteriormente suas avaliações são discutidas na Comissão Consultiva em Avaliação Psicológica (CCAP) formada por pesquisadores de diferentes partes do Brasil, especialistas na área.

Já internacionalmente, alguns países também realizam avaliações dos seus instrumentos. Uma edição especial da revista da International Test Comission, International Journal of Testing em 2012 (volume 12) publicou vários artigos sobre esta temática, apresentando a forma como é feita a avaliação em diversos países. Evers (2012) apresenta uma descrição breve sobre esses modelos. A seguir, abordarei algumas dessas formas de avaliação de acordo com buscas que realizei nos sites das diversas instituições.

Nos Estados Unidos, essa atividade é realizada pelo Buros Center for Testing, organização fundada por Oscar Buros, a partir da publicação do 1938 Mental Measurements Yearbook (disponível em https://buros. org/history). Nesse livro, Buros descreveu a análise que fez a partir da avaliação de diversos especialistas a respeito de vários testes de inteligência. Depois da morte de Buros em 1974, sua esposa continuou apoiando a organização até o momento em que, após uma disputa por diferentes universidades norte-americanas, o controle passou para as mãos da Universidade de Nebraska. Hoje seu diretor é o Prof. Kurt Geisinger, também presidente eleito da International Test Comission.

O Mental Measurements Yearbook foi sendo publicado em intervalos variados entre dois até nove anos e hoje está em sua vigésima edição. A cada edição, novos testes são avaliados por avaliadores ad-hoc. Foram avaliados 3.829 testes pelo Buros Center for Testing, entre os anos de 1985 e 2017 (da nona à vigésima edição), em uma média aproximada de 116 novos testes avaliados por ano (dados retirados do site http://buros.org/). A organização também possui uma nova publicação chamada Puebras Publicadas em Espanol, que revisou 583 testes em língua espanhola e que está na fase de editoração da segunda edição, da qual parecem muito orgulhosos (percepção que tive em recente visita ao Buros Center for Testing).

Nessa avaliação, os testes são distribuídos em níveis A, B e C, sendo que para cada nível é exigido um tipo de formação (aqui não me refiro somente ao curso de graduação, mas outras qualificações, como cursos e treina- mentos de educação continuada), ou seja, o profissional, ao adquirir um instrumento, deve comprovar qual é a sua formação ou treinamento. Por exemplo, para o nível A, não há qualquer exigência. Para o nível $\mathrm{B}$, exige-se que pelo menos o profissional possua treinamento em administração ética do instrumento, assim como levantamento e interpretação dos seus resultados, podendo ser de diferentes áreas (Psicologia, Educação, Terapia Ocupacional e outras). Já para o nível C, exige-se uma formação qualificada em interpretação de testes relacionados a sua área de formação. Quem faz o controle da venda são as editoras, orientadas pelas regras estabelecidas nos Standards for Educational and Psychological Testing (American Educational Research Association, American Psychological Association, \& National Council on Measurement in Education, 2014).

Outro país que avalia seus testes é a Espanha, por meio do Consejo General de Colegios Oficiales de Psicologos. Uma comissão de oito membros (um do conselho, um coordenador, um de cada uma das quatro editoras de testes reconhecidas pelo Conselho no país e dois de universidade) avaliam os instrumentos publicados no país. Essa avaliação ocorre desde 2012, e no total já foram avaliados 64 testes psicológicos. Uma rápida visão mostra que os instrumentos têm, em geral, notas altas. No site, não há referência a restrições de uso para os testes mal avaliados.

$\mathrm{Na}$ Espanha, os testes também são divididos em três níveis, tal como nos Estados Unidos. Para o nível A, espera-se formação e experiência em aplicação do instrumento de interesse. Para o nível B, conhecimentos sobre Psicometria e formação acadêmica correspondente ao instrumento de interesse. Somente para o nível C exige-se titulação em Psicologia, Psiquiatria ou Psicopedagogia (exemplo disso pode ser encontrado em: http://www.teaediciones.net/portal/recursos/disclaimer/index.html?seccion=normas\&header $=1$ ).

Na Grã-Bretanha, a British Psychological Society (BPS, disponível em www.psychtesting.org.uk) também avalia testes, os quais são submetidos de forma voluntária pelas editoras (em princípio, elas têm interesse nesse processo para demonstrar que seus testes são qualificados). Cento e sessenta testes já foram avaliados, por uma equipe de dois revisores independentes e duas editoras, utilizando os critérios criados pelo Grupo de Trabalho em Avaliação da Federação Europeia de Associações de Psicólogos, recentemente revisados (Evers et al., 2013). Os critérios são muito parecidos com os nossos do Satepsi. 
Além de avaliar os testes, a BPS possui um manual que explica quais são os requisitos que um profissional deve ter para utilizar testes psicológicos. Além disso, certifica profissionais por meio de três níveis diferentes de certificado: assistente de testagem, usuário de teste e especialista em uso do teste (só neste caso há referência ao profissional psicólogo, no qual afirmam que em geral é um psicólogo experiente, que fez já muitos cursos sobre testes e psicometria).

Na Holanda, os testes são avaliados pelo Comitê Holandês de Teste e Testagem do Instituto Holandês de Psicólogos desde 2009 (disponível em: https:// www.psynip.nl/en/dutch-association-psychologists/ activities-nip/psychological-testing-cotan/), chamado de Sistema Cotan. Os testes são avaliados com três objetivos: informar a qualidade do instrumento aos usuários, oferecer um feedback aos seus desenvolvedores e informar sobre a qualidade dos testes aos que estão sendo submetidos a ele. A avaliação não é cobrada nem dos autores do teste nem das editoras e os resultados de 841 testes já avaliados estão publicados online em holandês (https://www.cotandocumentatie.nl/). Eles possuem guias muito recentes (2017) de uso de testes, baseados em parte nas discussões da Federação Europeia de Associações de Psicólogos (EFPA). Essa sociedade também oferece um selo de Psicólogo NIP (ou seja, é reconhecido como um psicólogo especialista em alguma área).

Na Alemanha, existe uma norma chamada DIN ${ }^{\circ}$ 33.430 (Deutsches Institut fur Normung - Instituto Alemão de Padronização), que estabelece critérios para processos avaliativos das mais diversas áreas, assim como requisitos para um bom teste e uma boa testagem. É ressaltado que esta normatização não é somente voltada para testes psicológicos, mas sim qualquer forma de avaliação (mais detalhes em Hagemeister, Kersting, \& Stemmler, 2012). Os testes são revisados por um Conselho de Avaliação e Testagem da Associação Profissional de Psicólogos Alemães, chamado Sistema TBS-TK. As revisões são baseadas nas decisões da EFPA e do sistema Cotan (da Holanda) e são publicadas online. Até o momento (desde 2006), foram avaliados 38 testes (disponível em: http:/ /www.bdp-verband.de/ psychologie/testrezensionen/index.html).

Na África do Sul, os testes são avaliados desde 2015. Há um Comitê de Psicometria no Conselho dos Profissionais de Saúde da África do Sul que avalia os testes assim como estabelece normas de uso e construção dos mesmos (disponível em: http://
www.hpcsa.co.za/PBPsychology/RegulatingTestSA). Existe uma taxa de avaliação que é cobrada dos editores de testes quando os submetem para avaliação. Eles publicam uma lista com três categorias de testes: aqueles avaliados e certificados para uso por profissionais de Psicologia (total de 74 testes), aqueles liberados para uso por psicólogos, mas não avaliados (133) e aqueles que estão em processo de desenvolvimento ou adaptação (92) e que não devem ser utilizados para uso profissional (com intuito financeiro). Somente neste país ficou claro que alguns testes não são recomendados para uso profissional e que outros podem ser utilizados, mas somente por psicólogos. Para eles, usar uma medida, um teste, um questionário que avalie habilidades cognitivas ou intelectuais, atitudes, interesses, personalidade é considerado um ato psicológico a ser executado somente por um psicólogo registrado no conselho.

Como pode ser visto, a avaliação de instrumentos no mundo em geral é realizada por profissionais da área de Psicologia, pois é sempre realizada por profissionais vinculados a uma associação ou a um conselho profissional de Psicologia. Além disso, é uma atividade relativamente recente (com exceção dos Estados Unidos).

Quanto aos resultados da avaliação dos testes, podemos perceber que os mesmos são publicados de forma online em praticamente todos os países (por vezes, é cobrada uma taxa extra para um parecer mais detalhado). Essa atitude deixa o processo mais transparente, tentando evitar influência das editoras/autores dos testes nesse processo ou mesmo interesses financeiros ou de outra ordem.

Não foi possível obter a informação de que profissionais podem utilizar os instrumentos de dois países, Holanda e Alemanha. Porém, tendo em vista que se baseiam nas regulamentações da EFPA, tanto quanto a Sociedade Britânica, é de se entender que o que importa no uso é a formação do profissional, o conhecimento adquirido para o uso do instrumento. A África do Sul, assim como o Brasil, são os únicos países que restringem o uso dos testes somente para psicólogos registrados no conselho profissional.

\section{Certificação para o uso de testes e fiscalização}

A partir do exposto, entendo que para o uso de testes psicológicos, o que importa é o profissional ter uma formação adequada para cada o tipo de teste que 
ele queira utilizar. Essa formação deveria envolver aspectos básicos de psicometria, conhecimento teórico sobre o construto em questão e curso para aplicação, levantamento e interpretação, quando for o caso, seja ele psicólogo ou não.

Contudo, ainda assim continuaremos precisando que algum órgão faça a certificação e a fiscalização para uso dos testes. No meu entender, o CFP poderia seguir com esse papel, já que comparando o Satepsi com os modelos de avaliação de testes em outros países, estamos realizando um processo muito completo e bem qualificado. Talvez precisássemos reforçar dois aspectos. Um seria investirmos na publicação dessas avaliações, tornando esse processo mais transparente, protegendo e apoiando avaliadores e avaliados. O outro, termos regras claras de formação da equipe do Satepsi. Como ele está vinculado ao CFP, órgão que de dois em dois anos exige que os psicólogos votem para eleger sua diretoria, pode ser erroneamente utilizado com intenções políticas. A avaliação dos testes é uma atividade puramente técnica, jamais política. Por isso, deve haver uma legislação clara no sentido de que a comissão do Satepsi seja composta necessariamente por representantes das diversas entidades de avaliação psicológica no Brasil (Instituto Brasileiro de Avaliação Psicológica - IBAP, Associação Brasileira de Rorschach e Métodos Projetivos - Asbro, e Grupos de Trabalho da área de Avaliação Psicológica da Anpepp).

Assim como em outros países, penso que no Brasil os testes também poderiam ser classificados em níveis, exigindo diferentes tipos de formação. Esse nivelamento poderia ser realizado pelo próprio Satepsi. Poderia haver órgãos externos ao CFP, inclusive autorizados pelo CFP, tais como IBAP, Asbro, cursos de especialização reconhecidos, a emitir certificação para uso de instrumentos que exigissem maior formação, inclusive treinamento específico. Os profissionais teriam que comprovar seu conhecimento por meio de certificados de cursos realizados e com isso adquiririam esses selos. As editoras, por sua vez, poderiam vender seus instrumentos somente para aqueles que tivessem o selo.

A fiscalização seria, então, sobre a venda dos instrumentos aos profissionais com a formação adequada e/ou certificados por órgãos devidamente identificados pelo CFP. Atualmente, esse controle já é realizado pelo próprio CFP (conforme Resolução
CFP no 25/2001), ou seja, as editoras continuariam a ser reguladas.

\section{Considerações finais}

Tendo em vista o apontado, entendo que precisamos avançar mais um pouco nesse processo de qualificar os profissionais para o uso de instrumentos e realização de avaliações psicológicas com qualidade. Precisamos de psicólogos "válidos" (Bandeira, 2015), capazes de utilizar com propriedade os instrumentos que estão disponíveis para uso. Portanto, ou modificamos o curso e voltamos a dar total ênfase à avaliação psicológica ou criamos a especialidade em avaliação psicológica, dando mais qualidade a este trabalho.

Entendo, também, que os testes não são propriedades de um conselho ou de uma instituição. São de propriedade de uma editora ou de um autor, que pode ou não ser psicólogo. Acredito que se o autor foi capaz de construir um instrumento bem avaliado pelo Satepsi, possuindo ou não um construto psicológico, é capaz de o utilizar, mesmo não sendo um psicólogo. Ele é quem tem autoridade de afirmar que tipo de formação se espera da pessoa que usará seu teste.

Atualmente, há um contexto de formação profissional dinâmico, com fronteiras permeáveis e conhecimentos disponíveis na internet, de amplo acesso, totalmente diferente de alguns anos atrás. Com isso digo que, se não tomarmos a frente, seremos atropelados por essas questões, correndo o risco de perder a possibilidade que já temos de avaliar esses instrumentos.

Por meio de algumas ideias, tentei contribuir para a discussão sobre quem pode utilizar testes psicológicos. Concluo que para isso, o que importa mesmo é a formação do profissional, tendo ou não feito a graduação em Psicologia. Afirmo mais uma vez, nem todo o psicólogo no Brasil, só por possuir o número de registro no CFP, tem condições de utilizar testes psicológicos. E estamos permitindo isso!

Porém, como afirmei, são várias as questões envolvidas na pergunta pela qual inicio este artigo e vários desafios que precisam encarados para que possamos implementar mudanças com objetivo de qualificar nossa atuação na área de avaliação psicológica. Se tanto avançamos desde a criação da Câmara Interinstitucional de Avaliação Psicológica, pelo CFP, é porque a área já está madura para continuar avançando. 


\section{Referências}

Alchieri, J. C., \& Bandeira, D. R. (2002). O ensino da avaliação psicológica no Brasil. In: Ricardo Primi. (Org.), Temas em avaliação psicológica (pp. 35-39). São Paulo, SP: IBAP.

American Educational Research Association, American Psychological Association, \& National Council on Measurement in Education. (2014). Standards for educational and psychological testing. Washington, DC: American Educational Research Association.

Anache, A. A., \& Corrêa, F. B. (2010). As políticas do Conselho Federal de Psicologia para a avaliação psicológica. In Conselho Federal de Psicologia, Avaliação psicológica: diretrizes na regulamentação da profissão (pp. 19-30). Brasília. DF: o autor.

Bandeira, D. R. (2011). Repensando a formação em avaliação psicológica no Brasil. In Conselho Federal de Psicologia, Ano da avaliação psicológica: Textos geradores (pp. 129-132). Brasília, DF: o autor.

Bandeira, D. R. (2015). Prefácio. In S. M. Barroso, F. Scorsolini-Comin, \& E. Nascimento (Eds.), Avaliação psicológica: Da teoria às aplicações. Rio de Janeiro, RJ: Vozes.

Bandeira, D. R., Trentini, C. M., \& Krug, J. (2016). Psicodiagnóstico: formação, cuidados éticos, avaliação de demanda e estabelecimento de objetivos. In C. S. Hutz, D. R. Bandeira, C. M. Trentini, \& J. Krug, Psicodiagnóstico (pp. 21-26). Porto Alegre, RS: Artmed.

Conselho Federal de Psicologia - CFP. (2010). Avaliação psicológica: Diretrizes na regulamentação da profissão. Brasília, DF: o autor.

Conselho Federal de Psicologia - CFP. (2011). Ano da avaliação psicológica: Textos geradores. Brasília, DF: o autor.

Evers, A. (2012). The internationalization of test reviewing: Trends, differences, and results. International Journal of Testing, 12(2), 136-156. https:// doi.org/10.1080/15305058.2012.658932

Evers, A., Muñiz, J., Hagemeister, C., Høtmælingen, A., Lindley, P., Sjöbergr, A. et al. (2013). Assessing the quality of tests: revision of the EFPA review model. Psicothema, 25(3), 283-91. https://doi.org/10.7334/psicothema2013.97

Hagemeister, C., Kersting, M., \& Stemmler, G. (2012). Test reviewing in Germany. International Journal of Testing, 12(2), 185-194. https://doi.org/10.1080/15305058.2012.657922

Hutz, C. S. (2015). O que é avaliação psicológica: Método, técnicas e testes. In C. S. Hutz, D. R. Bandeira, \& C. M. Trentini. Psicometria (pp. 11-22). Porto Alegre, RS: Artmed.

Hutz, C. S., \& Bandeira, D. R. (2003). Avaliação psicológica no Brasil: Situação atual e desafios para o futuro. In O. H. Yamamoto, \& V. V. Gouveia (Org.). Construindo a psicologia brasileira: Desafios da ciência e prática psicológica (pp. 261-277). São Paulo, SP: Casa do Psicólogo.

Lei No 4.119, de 27 de agosto de 1962. Dispõe sobre os cursos de formação em Psicologia e regulamenta a profissão de psicólogo. Diário Oficial da União, 5 set. 1962.

Resolução No 05, de 15 de março de 2011. Institui as Diretrizes Curriculares Nacionais para os cursos de graduação em Psicologia, estabelecendo normas para o projeto pedagógico complementar para a Formação de Professores de Psicologia. Brasília, DF: Ministério da Educação.

Resolução No 009, de 25 de abril de 2018. Estabelece diretrizes para a realização de Avaliação Psicológica no exercício profissional da psicóloga e do psicólogo, regulamenta o Sistema de Avaliação de Testes Psicológicos - SATEPSI e revoga as Resoluções n 002/2003, no 006/2004 e n 005/2012 e Notas Técnicas nº 01/2017 e 02/2017. Brasília, DF: Conselho Federal de Psicologia.

Resolução $N^{\circ}$ 025, de 30 de novembro de 2001. Define teste psicológico como método de avaliação privativo do psicólogo e regulamenta sua elaboração, comercialização e uso. Brasília, DF: Conselho Federal de Psicologia.

Santos, A. A. A. (2011). O possível e o necessário no processo de avaliação psicológica. In: Conselho Federal de Psicologia. Ano da avaliação psicológica: Textos geradores (pp. 13-16). Brasília, DF: Conselho Federal de Psicologia.

Urbina, S. (2014). Essentials of psychological testing (2a ed.). Hoboken, NJ: John Wiley \& Sons. 


\section{Denise Ruschel Bandeira}

Professora do Programa de Pós-Graduação em Psicologia do Instituto de Psicologia da Universidade Federal do Rio Grande do Sul, Porto Alegre - RS. Brasil.

E-mail: bandeira@ufrgs.br

Endereço para envio de correspondência:

Instituto de Psicologia

Rua Ramiro Barcelos, 2600/120. CEP: 90035-003.

Porto Alegre - RS. Brasil.

Recebido: $20 / 07 / 2018$

Aprovado: 07/08/2018

Received:07/20/2018

Approved: 08/07/2018

Recibido:20/07/2018

Aceptado: 07/08/2018

Como citar: Bandeira, D. R. (2018). A controvérsia do uso dos testes psicológicos por psicólogos e não psicólogos. Psicologia: Ciência e Profissão, 38(n.spe), 159-166.. https://doi.org/10.1590/1982-3703000208860

How to cite: Bandeira, D. R.. (2018). The controversy of the use of psychological tests by psychologists and non psychologists. Psicologia: Ciência e Profissão, 38(n.spe), 159-166.. https://doi.org/10.1590/1982-3703000208860

Cómo citar: Bandeira, D. R. (2018). La controversia del uso de las pruebas psicológicas por psicólogos y no psicólogos. Psicologia: Ciência e Profissão, 38(n.spe), 159-166.. https://doi.org/10.1590/1982-3703000208860 